\title{
INTELLMANIPULATOR: MODELO COMPUTACIONAL BASEADO EM SISTEMAS MULTIAGENTE PARA AUTOMATIZAR OPERAÇÕES COM MANIPULADORES SUBAQUÁTICOS
}

\author{
Leonardo de Paula Nardy; Oberdan Rocha Pinheiro²; Herman Augusto Lepikson³ \\ 2Oberdan Rocha Pinheiro; Salvador/Bahia; oberdan.pinheiro@fieb.org.br \\ 1,3SENAI CIMATEC; Salvador/Bahia
}

Resumo: Este projeto de investigação propõe um modelo computacional baseado em sistemas multiagentes para automatizar operações com manipuladores subaquáticos. O desenvolvimento do modelo foi baseado na metodologia GAIA, utilizada em projetos de sistemas complexos baseados na abordagem multiagentes, uma proposta de arquitetura foi projetada para implementar e facilitar a utilização dos vários módulos do modelo computacional. Os resultados preliminares indicam que deve ser melhorada a otimização dos algoritmos em referência ao tempo de distribuição da lista local dos agentes. Espera-se que esta plataforma facilite o desenvolvimento de novas metodologias relativas a automatização de operações com os manipuladores subaquáticos.

Palavras-Chave: Sistemas Muitlagentes; Manipuladores; ROV.

\section{INTELLMANIPULATOR: DEVELOPMENT OF INTELLIGENT UNDERWATER MANIPULATORS BASED ON MULTIAGENT SYSTEMS}

\begin{abstract}
This research project proposes a computational model based on multi-agent systems to automate operations with underwater manipulators. The development of the model was based on the GAIA methodology, used in complex system projects based on the multi-agent approach. An architectural proposal was designed to implement and facilitate the use of the various modules of the computational model. Preliminary results indicate that the optimization of algorithms in reference to the distribution time of the local agent list should be improved. This platform is expected to facilitate the development of new methodologies for automating operations with underwater handlers.
\end{abstract}

Keywords: Multiagent systems; Manipulators; ROV. 


\section{INTRODUÇÃO}

Devido as grandes distâncias entre os equipamentos no fundo do mar e as plataformas de petróleo, e sabendo-se que a presença humana por meio do trabalho de mergulhadores está limitada à profundidade de 300 metros, as atividades e operações que excedem essa profundidade são possíveis com o apoio de Veículos Operados Remotamente (ROV). As empresas do setor de petróleo, gás natural, energia e biocombustíveis, tem grande interesse em realizar intervenção submarina utilizando manipuladores subaquáticos, com eficiência e precisão em lugares onde a presença humana torna-se difícil, arriscada ou até mesmo impossível. Para solucionar este problema, é necessário o emprego de mecanismos autônomos ou remotos que substituam os seres humanos na execução dessas tarefas.

As operações com ROV normalmente exige uma equipe offshore formada por 3 (três) profissionais especializados, sendo: um observador, um operador do braço manipulador e um navegador. Esta equipe muitas vezes precisa ser duplicada devido aos turnos de trabalho, pois as missões de intervenção submarina com ROV demanda uma quantidade significativa de controle manual de baixo nível do manipulador, elevando os custos operacionais com tarefas típicas envolvidas nas missões que exigem grande destreza, como, por exemplo: instalação e manutenção de Árvore de Natal Molhada (ANM), um dos equipamentos responsáveis pela produção de petróleo. A automatização das tarefas envolvidas nessas missões pode aumentar a eficiência do processo e, consequentemente, reduzir os custos de operação e o tempo de comissionamento. A Figura 1 ilustra uma missão de intervenção em uma ANM por uma equipe a partir de uma sala de controle na embarcação de apoio. O ROV conta com dois manipuladores subaquáticos, sendo um deles (4 graus de liberdade) utilizado para ancoragem do ROV e outro (6 graus de liberdade) para realizar as operações de intervenção. O operador do manipulador dispõe de um joystick para realizar o controle do manipulador, girando-o de um lado para outro e/ou para trás e frente, baseados em imagens $2 \mathrm{D}$, obtidas por câmeras de vídeo e transmitidas através de umbilicais. O sucesso da missão depende da experiência do operador.

Figura 1. ROV em intervenção subaquática
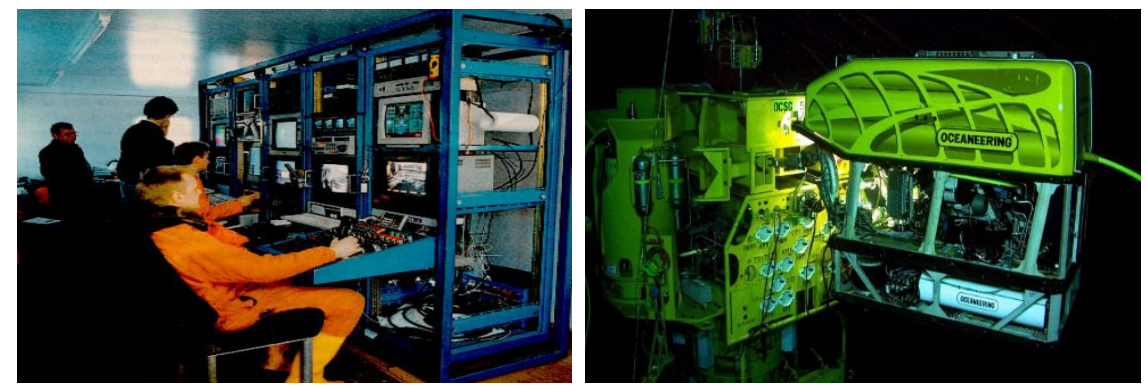

Este trabalho de pesquisa propõe um modelo computacional genérico e modular para desenvolvimento de manipuladores subaquáticos inteligentes baseado em sistemas multiagentes (SMA), denominado IntellManipulator. O projeto aborda o estudo de uma plataforma que permita facilitar o desenvolvimento de sistemas sensoriais e de atuação e de novas metodologias de planeamento inteligente voltados 
para as operações envolvendo manipuladores subaquáticos. Além disso, concentrase em tornar as operações autônomas uma opção viável nesse setor, reduzindo os custos operacionais, aumentando a segurança das missões e apoiando a expansão do setor offshore. O restante do artigo está organizado da seguinte forma: a Seção 2 descreve os sistemas de controle de manipuladores subaquáticos; a seção 3 apresenta o conceito do projeto IntellManipulator; na seção 4 são apresentados os cenários de teste e os resultados da avaliação; a seção 5 apresenta as conclusões e continuidade do projeto.

\section{SISTEMAS DE CONTROLE DE MANIPULADORES SUBAQUÁTICOS}

Manipuladores subaquáticos comerciais acoplados aos ROVs são controlados por sistemas remotos e são totalmente dependentes do operador. Com o objetivo de melhorar 0 desempenho dos manipuladores em operações subaquáticas, pesquisadores propuseram melhorias no nível cinemático. Alguns se concentraram na melhoria das técnicas convencionais de teleoperação, enquanto outros recorrem à utilização de técnicas de controle semiautônomas e autônomas, na esperança de transferir o controle para uma posição de supervisão. Algumas das pesquisas iniciais sobre aprimoramentos de controle de teleoperação incluem o desenvolvimento de uma interface homem-máquina onde uma variedade de possíveis dispositivos de entrada de controle remoto está disponível, incluindo teclado, mouse, braço mestre e/ou joystick, cada um dos quais fornece benefícios para diferentes tarefas [1]. Outra abordagem importante que tem sido investigada por muitos pesquisadores inclui simulações de movimento em tempo real e reconstrução virtual do espaço de trabalho do manipulador, incluindo modelos das peças de trabalho apresentados na tela do monitor. Tais ferramentas fornecem complementos visuais à teleoperação, ajudando o operador a perceber a pose do manipulador subaquático e possivelmente evitar a colisão com outros objetos. Pesquisas pioneiras sobre este tema são relatadas em [2, 3]. [4] apresentou soluções baseadas no controle da cinemática inversa levando em consideração o custo computacional, a solução foi aplicada para controlar um manipulador subaquático comercial, um Schilling Orion de 6 graus de liberdade. Em [5] foi desenvolvido um sistema de controle espacial cartesiano com a implementação de uma versão modificada do ECA ARM 5E. [6] utilizaram uma solução analítica de cinemática inversa para o manipulador subaquático de seis graus de liberdade desenvolvido pelo Instituto de Automação de Shenyang da Academia Chinesa de Ciências. Em [7] foi apresentado um método numérico para resolver o problema da cinemática inversa para um manipulador subaquático comercial, Schilling Titan 2. 0 projeto PANDORA explorou o aprendizado da trajetória do veículo e do manipulador por demonstração para realizar o torneamento de válvulas [8]. No projeto MERBOTS, [9] utilizou o pacote de planejamento de movimento ROS Movelt para calcular trajetórias. Problemas avançados de controle considerando manipuladores subaquáticos são apresentados em [10-13].

Embora existam projetos de pesquisa na busca pela automatização de operações de ROV com manipuladores subaquáticos (e até alguns testados em manipuladores comerciais), verifica-se a inexistência de uma plataforma com reais 
capacidades de controle inteligente de ações autônoma. Torna-se assim cada vez mais necessário dotar manipuladores subaquáticos de mecanismos que permitam realizar de forma autônoma a execução de missões, através de metodologias como: linguagens de comando de alto nível, planejamento inteligente de ações, comunicação com outros dispositivos inteligentes, baseado no paradigma SMA nestes ambientes. Da análise realizada dos principais projetos de investigação, pode-se verificar a inexistência de um projeto capaz de adaptar um manipulador subaquático transformando-o em um manipulador inteligente. Para tal seria necessário realizar adaptação com o princípio de causar o menor impacto sobre o aspecto original do sistema, com modularidade de hardware que permita sua utilização em diferentes modelos com mínimas modificações e com uma plataforma de software que permita sua interação em ambiente simulado. Este trabalho procura preencher algumas destas lacunas, neste sentido foca-se na criação de uma ferramenta que ajude a desenvolver equipamentos que atendam estas necessidades.

\section{METODOLOGIA}

O desenvolvimento do projeto foi baseado na metodologia GAIA [14]. Trata-se de uma metodologia aberta para desenvolvimento de sistemas complexos baseados na abordagem multiagente. A Figura 2 apresenta a arquitetura proposta do projeto IntellManipulator.

Figura 2. Arquitetura do projeto

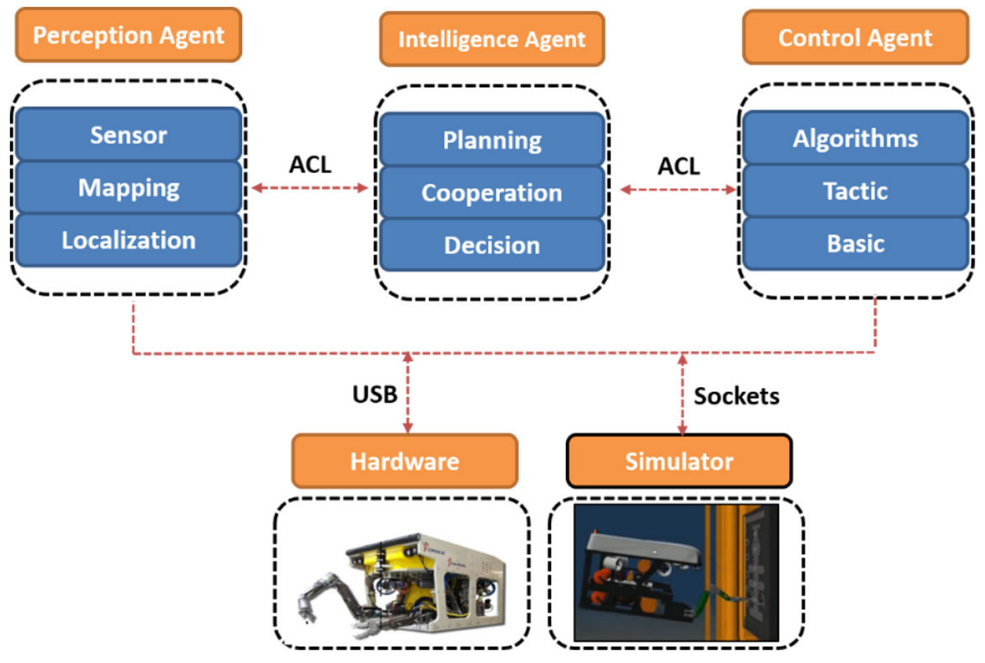

A arquitetura foi projetada para implementar e facilitar a utilização de vários módulos de forma independente. Os agentes projetados foram:

- Perception Agent - Representa o sistema de percepção, responsável pela leitura dos dados do sensor e atualização do ambiente interno, mapeamento e localização. 
- Intelligence Agent - Responsável pelas decisões de alto nível, assim como, monitorar em tempo real a execução do plano de trabalho (objetivo da missão) e a cooperação com outros agentes.

- Control Agent - Implementa a camada de controle do manipulador subaquático, com funções especificas, com por exemplo: seguir uma trajetória linear, mover-se até um ponto $(x, y, z)$ e acionar ferramenta de trabalho.

A plataforma multiagente Java Agent Development Framework (JADE) [15], foi utilizada na implementação do sistema de comunicação. Adotou-se as diretrizes da Foundation for Intelligent Physical Agents (FIPA) [16], assim como a linguagem de comunicação Agent Communication Language (ACL). A solução adotada contempla um conjunto de tecnologias para que os agentes possam interagir com outros agentes escritos em diferentes linguagens de programação executados em outras plataformas.

\section{RESULTADOS E DISCUSSÃO}

Para avaliar e validar o funcionamento da arquitetura proposta, foi criado o cenário de testes de escalabilidade da plataforma. O objetivo foi avaliar o desempenho dos agentes na plataforma JADE. Foi implementado um agente de controle e alguns agentes de percepção e inteligência. $O$ teste foi configurado dessa forma, porque o agente Control é o que demanda mais interações, sendo responsável por iniciar as operações. O tempo necessário para execução de uma ação (incluindo pedido e resposta) foi obtido em cinco repetições, no final foi considerado o valor médio. A Figura 3 apresenta os valores obtidos nos testes.

Figura 3. Tempo para execução completa de um pedido de controle

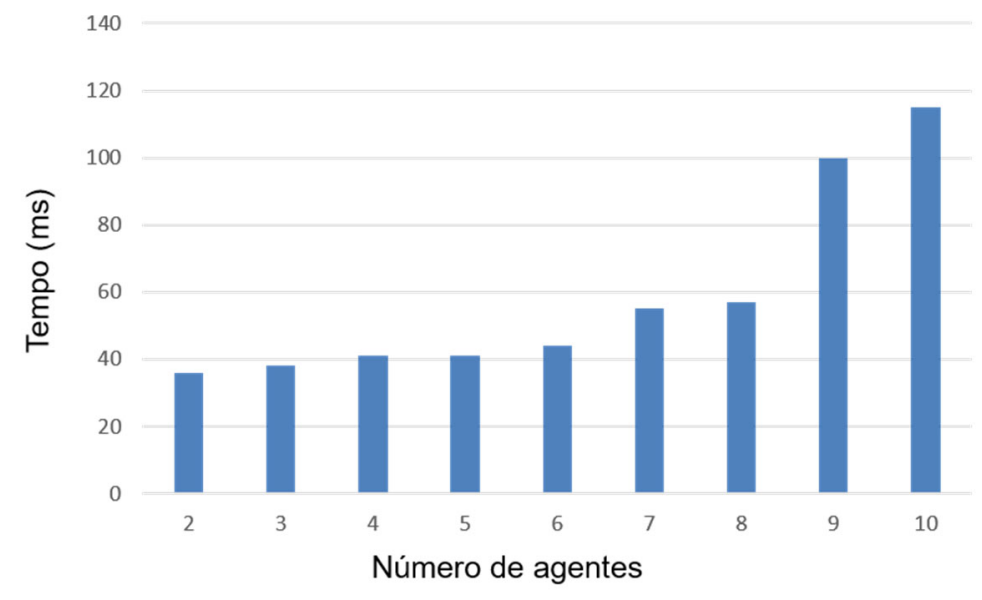

É possível observar a partir dos dados (Figura 3) que o sistema começa a exigir mais tempo para executar uma interação a partir de 9 agentes no ambiente. Uma característica do ambiente subaquático envolvendo operações com ROV e 
manipuladores, é o fato de existir pequenas perturbações provocadas pelas correntes marítimas. Esse fato exige do sistema respostas rápidas para adaptar-se as mudanças e atualizar o mapa interno do sistema, permitindo aos agentes reprogramar as ações para atingir o objetivo final da missão. Nesse sentido, foi investigado as características da plataforma JADE relacionadas a gestão dos agentes. Foi identificado que a plataforma mantém duas listas com informações dos agentes (local e global). A lista global armazena o estado dos agentes remotos, já a lista local armazena dados dos agentes residentes na máquina onde o sistema está em execução. Nesse sentido, foi criado um novo cenário de testes com o objetivo de avaliar o tempo necessário para criação e distribuição da lista local, já que os agentes são executados na mesma máquina. A Figura 4 apresenta os tempos para criar e distribuir a lista local entre os agentes.

Figura 4. Tempo para criação e distribuição da lista local

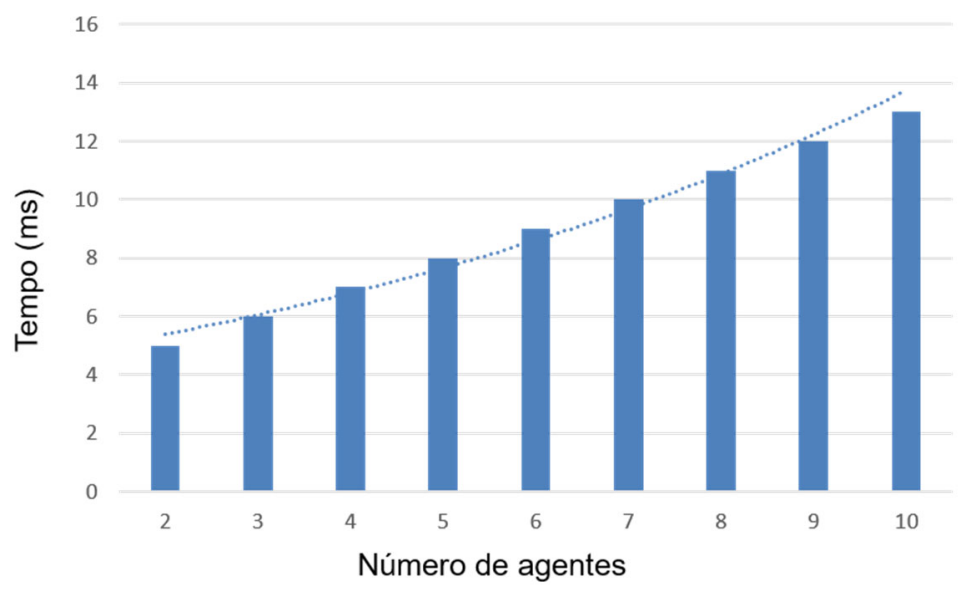

Ao analisar a linha de tendência (Figura 4), observa-se um comportamento exponencial na medida em que se aumenta os agentes. Esse comportamento, ocorre, pois, toda vez que é inserido um novo agente, o container precisa atualizar a lista local e enviar a todos os agentes. Estes resultados motivaram uma mudança no algoritmo de distribuição da lista local que está em desenvolvimento.

\section{CONCLUSÃO}

O projeto tem foco na construção de uma plataforma multiagente, que permita a fácil integração de diferentes sistemas de controle para resolver problemas associados a manipuladores subaquáticos inteligentes. Espera-se que esta plataforma facilite o desenvolvimento novas metodologias relativas aos manipuladores subaquáticos inteligentes. Dentro deste conceito, busca-se criar um conjunto de tecnologias (software e hardware) que possa ser facilmente integrado em qualquer sistema de controle de manipuladores subaquáticos, disponível comercialmente, com pequenas modificações. Os resultados dos testes mostram que - sistema de comunicação baseado na arquitetura proposta pode fornecer comunicação e cooperação entre agentes. No entanto, os dados observados nos 
testes indicam que deve ser melhorada a otimização dos algoritmos em referência ao tempo de distribuição da lista local dos agentes, pois, para um elevado número de agentes, os tempos de manutenção da plataforma começam a aumentar exponencialmente. Devido ao avanço nos projetos envolvendo automação de operações com manipuladores subaquáticos, deve ser dada atenção aos sistemas de comunicação. Neste campo, a interação com outros dispositivos é necessária, de modo a permitir a cooperação/colaboração entre os equipamentos existentes em um ambiente dito inteligente.

Como continuidade do desenvolvimento do projeto, são listados recursos que poderão servir de ponto de partida para desenvolvimento da plataforma, a saber: finalizar o algoritmo de manutenção da lista local; desenvolver os módulos dos agentes de percepção, controle e inteligência; adaptar o sistema levando em consideração as normas para sistemas críticos operando em águas profundas. $O$ desenvolvimento das funcionalidades sugeridas levará à criação de um manipulador subaquático inovador com elevadas capacidades no auxilio as operações envolvendo ROVs e com potencial para ser transformado em um produto comercial.

\section{REFERÊNCIAS}

${ }^{1}$ Larkum and Broome, "Advanced controller for an underwater manipulator," 1994 Proceedings of IEEE International Conference on Control and Applications, Glasgow, UK, 1994, pp. 1081-1086 vol.2. doi: 10.1109/CCA.1994.381364.

2 M. Hildebrandt, J. Kerdels, J. Albiez, F. Kirchner Robust vision-based semiautonomous underwater manipulation. Proceedings of the 10th International Conference on Intelligent Autonomous Systems (2008), pp. 308-315.

${ }^{3}$ S. Sivčev, M. Rossi, J. Coleman, G. Dooly, E. Omerdić, D. Toal Fully automatic visual servoing control for work-class marine intervention ROVs. Contr. Eng. Pract., 74 (2018), pp. 153-167.

${ }^{4}$ J. Albiez, M. Hildebrandt, J. Kerdels, F. Kirchner Automatic workspace analysis and vehicle adaptation for hydraulic underwater manipulators. Proceedings of the OCEANS 2009, ISSN 0197-7385 (2009), pp. 1-6.

5 J.J. Fernandez, M. Prats, P.J. Sanz, J.C. Garcia, R. Marin, M. Robinson, D. Ribas, P. Ridao Grasping for the seabed: developing a new underwater robot arm for shallowwater intervention. IEEE Robot. Autom. Mag., 20 (4) (2013), pp. 121-130 ISSN 10709932. 
${ }^{6}$ L. Huo, Q. Zhang, Z. Zhang A method of inverse kinematics of a 7-function underwater hydraulic manipulator. Proceeedings of the 2013 OCEANS - San Diego (2013), pp. 1-4 ISSN 0197-7385.

${ }^{7}$ S. Sivčev, J. Coleman, D. Adley, G. Dooly, E. Omerdié, D. Toal Closing the gap between industrial robots and underwater manipulators. Proceedings of the OCEANS 2015-MTS/IEEE Washington (2015), pp. 1-7.

${ }^{8}$ A. Carrera, N. Palomeras, D. Ribas, P. Kormushev, M. Carreras An Intervention-AUV learns how to perform an underwater valve turning. OCEANS 2014-TAIPEI (2014), pp. $1-7$.

${ }^{9}$ D. Youakim, P. Ridao, N. Palomeras, F. Spadafora, D. Ribas, M. Muzzupappa Movelt!: autonomous underwater free-floating manipulation. IEEE Robot. Autom. Mag., 24 (3) (2017), pp. 41-51 ISSN 1070-9932.

10 Multiple Impedance Control for object manipulation by a dual arm underwater vehicle-manipulator system. Ocean. Eng., 89 (2014), pp. 82-98 ISSN 0029-8018.

${ }^{11}$ D. Lillo, P. Augusto, E. Simetti, D. De Palma, E. Cataldi, G. Indiveri, G. Antonelli, G. Casalino Advanced ROV autonomy for efficient remote control in the DexROV project Mar. Technol. Soc. J., 50 (4) (2016), pp. 67-80.

12 H. Farivarnejad, S.A.A. Moosavian Multiple Impedance Control for object manipulation by a dual arm underwater vehicle-manipulator system. Ocean. Eng., 89 (2014), pp. 82-98 ISSN 0029-8018.

${ }^{13}$ R.B. Ambar, S. Sagara, K. Imaike Experiment on a dual-arm underwater robot using resolved acceleration control method. Artif. Life Robot., 20 (1) (2015), pp. 34-41.

${ }^{14}$ Wooldridge, M., Jennings, N.R. \& Kinny, D., 2000. The Gaia Methodology For AgentOriented Analysis And Design. Journal of Autonomous Agents and Multi-Agent Systems, September. pp.285-312.

${ }^{15}$ Bellifemine, F.L., Caire, G. \& Greenwood, D., 2007. Developing multi-agent systems with JADE. 1st ed. Chichester, England: Wiley.

${ }^{16}$ FIPA, 2009. FIPA, Foundation for Intelligent Physical Agents, online. Disponível em: http://www.fipa.org. 